\title{
Plasma Levels of Alpha and Gamma Synucleins in Autism Spectrum Disorder: An Indicator of Severity
}

\author{
Sarah Al-Mazidi ${ }^{a} \quad$ Laila Y. Al-Ayadhi $^{\text {b, c }}$ \\ aDepartment of Physiology, Faculty of Medicine, Imam Mohammed bin Saud Islamic University, Riyadh, Saudi \\ Arabia; ${ }^{b}$ Autism Research \& Treatment Center, Al-Amodi Autism Research Chair, Faculty of Medicine, King Saud \\ University, Riyadh, Saudi Arabia; ' Department of Physiology, Faculty of Medicine, King Saud University, Riyadh, \\ Saudi Arabia
}

\section{Highlights of the Study}

- Plasma levels of $\alpha$-synuclein and $\gamma$-synuclein were correlated with the severity of autism spectrum disorder (ASD).

- Plasma levels of $\alpha$-synuclein were higher and those of $\gamma$-synuclein were lower in children with ASD than normal children.

- $\alpha$-synuclein and $\gamma$-Synuclein were significantly correlated with the severity of ASD.

\section{Keywords}

Autism spectrum disorder · Neurology · Physiology ·

Psychiatry

\begin{abstract}
Objectives: The aim of this study was to correlate plasma levels of the synaptic proteins $\alpha$-synuclein and $\gamma$-synuclein in autism spectrum disorder (ASD) children in order to elucidate their possible contribution to the pathogenesis of ASD and to study their association with the severity of the disorder. Subjects and Methods: Plasma levels of a-synuclein and $Y$-synuclein were measured in 38 male children diagnosed with ASD and 40 healthy age-matched male children by ELISA. Results: Our results showed that plasma levels of a-synuclein (18.02 $\pm 5.3 \mathrm{pg} / \mathrm{mL})$ were significantly higher in ASD children than in control children $(14.39 \pm 2 \mathrm{pg} / \mathrm{mL})$, and
\end{abstract}

karger@karger.com www.karger.com/mpp

Karger $\stackrel{\text { ' }}{5}$

BOPEN ACCESS
(C) 2020 The Author(s)

Published by S. Karger AG, Basel

This is an Open Access article licensed under the Creative Commons Attribution-NonCommercial-4.0 International License (CC BY-NC) (http://www.karger.com/Services/OpenAccessLicense), applicable to the online version of the article only. Usage and distribution for commercial purposes requires written permission. plasma levels of $\mathrm{Y}$-synuclein were decreased in the ASD group $(23.74 \pm 7.7 \mathrm{pg} / \mathrm{mL}$ ) compared to the control group $(32.40 \pm 6.8 \mathrm{pg} / \mathrm{mL})(p<0.0001)$. Our data also indicate that plasma levels of both a-synuclein and $\gamma$-synuclein are significantly associated with the severity of ASD. Conclusions: Our study showed that alteration in a-synuclein and $\gamma$-synuclein might be associated with ASD pathogenesis and could be an indicator of the severity of the disorder.

(C) 2020 The Author(s)

Published by S. Karger AG, Basel

\section{Introduction}

Autism spectrum disorder (ASD) is a complex neurodevelopmental disorder characterized by communication impairment, and repetitive and stereotyped patterns of behaviors [1]. The prevalence of ASD is increasing rap- 
idly in the past decade, and it can differ according to the geographic area due to many factors but, most importantly, the socioeconomic factors that lead to misdiagnosis of ASD [2]. The etiology of ASD is heterogonous and complex. The pathogenesis of ASD involves multiple factors, including genetic, environmental, immune dysregulation, neuroinflammation, and oxidative stress [1]. Synaptic imbalance and mutation in synaptic proteins and receptors have also been reported in patients with ASD [3].

Synucleins are a family of small soluble proteins that are expressed abundantly in presynaptic terminals. Smaller amounts are found in other tissues like RBCs, heart, and muscles. They have an important role in regulating synaptic plasticity and neurotransmitters. The synuclein family consists of synuclein $\alpha$, synuclein $\beta$, and synuclein $\gamma$; all of these have been found to be important in the presynaptic terminal topography [4]. They are abundantly expressed in the brain and neurons. The structural properties of $\gamma$ - and $\alpha$-synuclein are similar, while the $\beta$-synuclein structure was found to be different and aggregates less than $\gamma$ - and $\alpha$-synuclein. Also, $\beta$-synuclein has an inhibitory effect on both $\gamma$ - and $\alpha$-synuclein activities [5]. $\alpha$-synuclein was extensively studied in neurodegenerative diseases, while little is known about the association of $\beta$ - and $\gamma$-synucleins in neurodegenerative diseases.

a-synuclein is known to be associated with many neurodegenerative disorders that are collectively called synucleinopathies. These disorders include Alzheimer's disease (AD), Parkinson's disease (PD), dementia with Lewy bodies (DLBs), and multiple system atrophy. In these diseases, $\alpha$-synuclein overexpression and $\backslash$ or misfolding leads to protein aggregation and the formation of amyloid fibril in Lewy bodies which is a hallmark in PD [6]. Another study reported that $\alpha$-synuclein and $\gamma$-synuclein, but not $\beta$-synuclein, affect the risk of developing diffuse Lewy body disease [7].

In addition, $\alpha$-synuclein has been reported to inhibit certain signaling pathways of brain-derived neurotrophic factor (BDNF), leading to dopaminergic neuronal death, which causes neurotoxicity in PD [8]. Also, the overexpression of $\alpha$-synuclein and $\gamma$-synuclein is associated with cognitive deficits $[9,10]$.

A recent study reported a strong association between neurodegenerative and neurodevelopmental disorders, specifically PD and ASD [11]. This association includes genetic, molecular, and even clinical similarities between these disorders.

Due to this association between the above disorders and the structural similarity between $\alpha$-synuclein and $\gamma$-synuclein, we investigated plasma levels of $\alpha$-synuclein and $\gamma$-synuclein in ASD children to demonstrate their possible contribution to the pathogenesis of ASD. We also studied the association of $\alpha$-synuclein and $\gamma$-synuclein with the severity of the disorder. This study is the first to examine the correlation of $\gamma$-synuclein in ASD children and the severity of ASD symptoms with $\gamma$-and $\alpha$-synuclein.

\section{Subjects and Methods}

\section{Subjects}

The ASD group included 38 male children with a confirmed diagnosis of ASD aged 2-12 years who fulfilled this study's inclusion criteria. They were recruited from the Autism Research and Treatment Center, Faculty of Medicine, King Saud University. The diagnosis of autism in all ASD patients was based on the Diagnostic and Statistical Manual of Mental Disorders, Fourth Edition (DSM-IV). The control group included 40 age-matched healthy male children who attend the pediatrics clinic of King Khalid University Hospital for routine follow-up. Any participant with an infectious disease or with neuropsychiatric disorders was excluded from the study. Informed consent forms were obtained from all participants' parents as approved by the Institutional Review Board of the Faculty of Medicine, King Saud University, according to the most recent Declaration of Helsinki.

\section{Behavioral Assessment}

Childhood Autism Rating Score

The childhood autism rating score (CARS) is an observational scale of 15 items used to distinguish children with ASD from other types of delays. The CARS assesses the child on a scale from 1 (normal) to 4 (severe abnormality) in the following 15 items: relating to people, emotional response, imitation, body use, object use, listening response, fear or nervousness, verbal communication, nonverbal communication, activity level, level and consistency of intellectual response, adaptation to change, visual response, taste, smell and touch response, and general impressions. According to the scale, children who score between 30 and 36 have mild to moderate autism, while those with scores between 37 and 60 points have severe autism.

\section{Social Responsiveness Scale}

The social responsiveness scale (SRS)is a 65 -item quantitative questionnaire that measures the severity of autistic behaviors and is completed in 15-20 min using a " 0 " (not true) to " 3 " (almost always true) scale. It focuses on the child's behavior during the previous 6 months. Children were rated in 5 subscale areas: social awareness, social cognition, social communication, social motivation, and autistic mannerisms. An SRS score between 60 and 75 is considered a mild to moderate range of social impairment. In contrast, a 76 or higher score is considered as severe and is strongly associated with a clinical diagnosis of ASD.

Short Sensory Profile

The short sensory profile (SSP)is a 38-item questionnaire used to rate a variety of sensory impairments. Each item on the SSP is measured on a 5 -point Likert scale ( 1 being "Always" and 5 being 
"Never"). Domain scores are measured in the areas of tactile sensitivity, taste/smell sensitivity, movement sensitivity, seeking sensation, auditory filtering, low-energy levels, and visual/auditory sensitivity. Lower scores indicate a more significant association with ASD. Domain scores and the overall sensory response are categorized as follows: $<142$ indicate severe performance (definite difference from typical performance), scores between 142 and 152 indicate mild to moderate performance (probable difference from typical performance), and scores between 153 and 190 indicate typical performance.

Measurement of Plasma $\alpha$-Synuclein and $\gamma$-Synuclein

Five milliliters of blood was collected in EDTA tubes from each subject in both groups. Samples were centrifuged to isolate the plasma, which was aliquoted and stored at $-80^{\circ} \mathrm{C}$ until analysis. Plasma concentrations of $\alpha$-synuclein and $\gamma$-synuclein were measured by ELISA using a commercial kit according to the manufacturer' instructions (human $\alpha$-synuclein assay, $\gamma$-synuclein assay, ELISA kit, Wuhan EIAab Science Company, Wuhan, China). Samples and standards were added to the precoated plate with antibodies specific for $\alpha$-synuclein or $\gamma$-synuclein and analyzed in duplicates for each protein of interest.

\section{Statistical Analysis}

The data are presented as mean \pm standard deviation. Data were statistically analyzed with one-way repeated-measures ANOVA. Bonferroni correction was used to compare the levels of $\alpha$-synuclein and $\gamma$-synuclein in the subcategories (mild-moderate, severe) of CARS, SSP, and SRS. Data that were not normally distributed (Shapiro-Wilk normality test) are presented as median and statistical analysis was performed with ANOVA, followed by Dunnett's post hoc test to compare $\alpha$-synuclein and $\gamma$-synuclein levels in all subgroups. Student's $t$ test was also used for comparing the plasma levels of $\alpha$-synuclein and $\gamma$-synuclein in ASD and control groups. Statistical analysis was performed with GraphPad Prism software, version 5 (GraphPad, San Diego, CA, USA). Twotailed tests for statistical significance were performed where $p<$ 0.05 was considered significant. Statistical significance was denoted as ${ }^{*} p<0.05 ;{ }^{* *} p<0.01 ;{ }^{* * *} p<0.001$.

\section{Results}

\section{Demographic Data}

The demographic and clinical data of ASD patients and healthy controls are shown in Table 1. The ASD group consisted of 38 autistic boys aged 2-12 years with a mean age of $5.0 \pm 2.5$ years, while the healthy control group consisted of 40 age-matched healthy boys with a mean age of $5.8 \pm 1.8$ years.

\section{Plasma Levels of $\alpha$-Synuclein and $\gamma$-Synuclein in ASD and Control Groups}

Plasma levels of $\alpha$-synuclein ranged between 12 and 40 $\mathrm{pg} / \mathrm{mL}$ in the ASD group and between 9 and $19 \mathrm{pg} / \mathrm{mL}$ in the control group. As shown in Figure 1a, plasma levels
Table 1. Demographic and clinical characteristics of the ASD and control groups

\begin{tabular}{|c|c|c|c|}
\hline Variable & $\begin{array}{l}\text { ASD } \\
(\text { mean } \pm \text { SD })\end{array}$ & $\begin{array}{l}\text { Control } \\
(\text { mean } \pm \mathrm{SD})\end{array}$ & $p$ value \\
\hline Age & $5.026 \pm 2.5$ & $5.8 \pm 1.8$ & $>0.05$ \\
\hline CARS & $36.16 \pm 3.7$ & - & - \\
\hline SRS & $79.97 \pm 8.4$ & - & - \\
\hline SSP & $109 \pm 36.2$ & - & - \\
\hline \multicolumn{4}{|c|}{ Laboratory findings (mean \pm SD) } \\
\hline a-Synuclein, pg/mL & $18.02 \pm 5.3$ & $14.39 \pm 2$ & $<0.001$ \\
\hline$\gamma$-Synuclein, pg/mL & $23.74 \pm 7.7$ & $32.40 \pm 6.8$ & $<0.0001$ \\
\hline
\end{tabular}

ASD, autism spectrum disorder; SD, standard deviation; CARS, childhood autism rating score; SSP, short sensory profile; SRS, social responsiveness scale.

of $\alpha$-synuclein were significantly higher in the ASD group than in the control group $(p<0.0001)$. The plasma levels of $\gamma$-synuclein ranged between 21 and $44 \mathrm{pg} / \mathrm{mL}$ in the ASD group and between 22 and $49 \mathrm{pg} / \mathrm{mL}$ in the control group. Figure $1 \mathrm{~b}$ shows that plasma levels of $\gamma$-synuclein were decreased significantly in the ASD group compared to the control group $(p<0.0001)$.

\section{Correlation between Age and Levels of $\alpha$-Synuclein and $\gamma$-Synuclein}

Figure $2 \mathrm{a}$ and $\mathrm{b}$ shows that the ASD and control groups were divided into subgroups as follows: ages 2-4, ages $5-7$, and ages $0-12$. Plasma levels of $\alpha$-synuclein were significantly increased in the 2-4 years control subgroup compared to its age-matched ASD group $(p<0.01)$. It also shows that plasma levels of $\gamma$-synuclein in the 2-4 years and 5-7 years subgroups are significantly decreased in children with ASD compared to children in the control group $(p<0.05)$. Also, $\gamma$-synuclein was significantly increased in the ASD group in the 10-12 years subgroup compared to the ASD group in both 2-4 years and 5-7 years subgroups. This indicates that age is associated with $\gamma$-synuclein levels in ASD patients.

\section{Comparison of Levels of $\alpha$-Synuclein and $\gamma$-Synuclein} in ASD Subgroups according to Their CARS, SRS, and SSP Scores

Plasma levels of $\alpha$-synuclein and $\gamma$-synuclein were compared between ASD subgroups with different degrees of severity and with the control group. Data were presented as mean \pm standard deviation. To evaluate whether $\alpha$-synuclein and/or $\gamma$-synuclein are correlated 


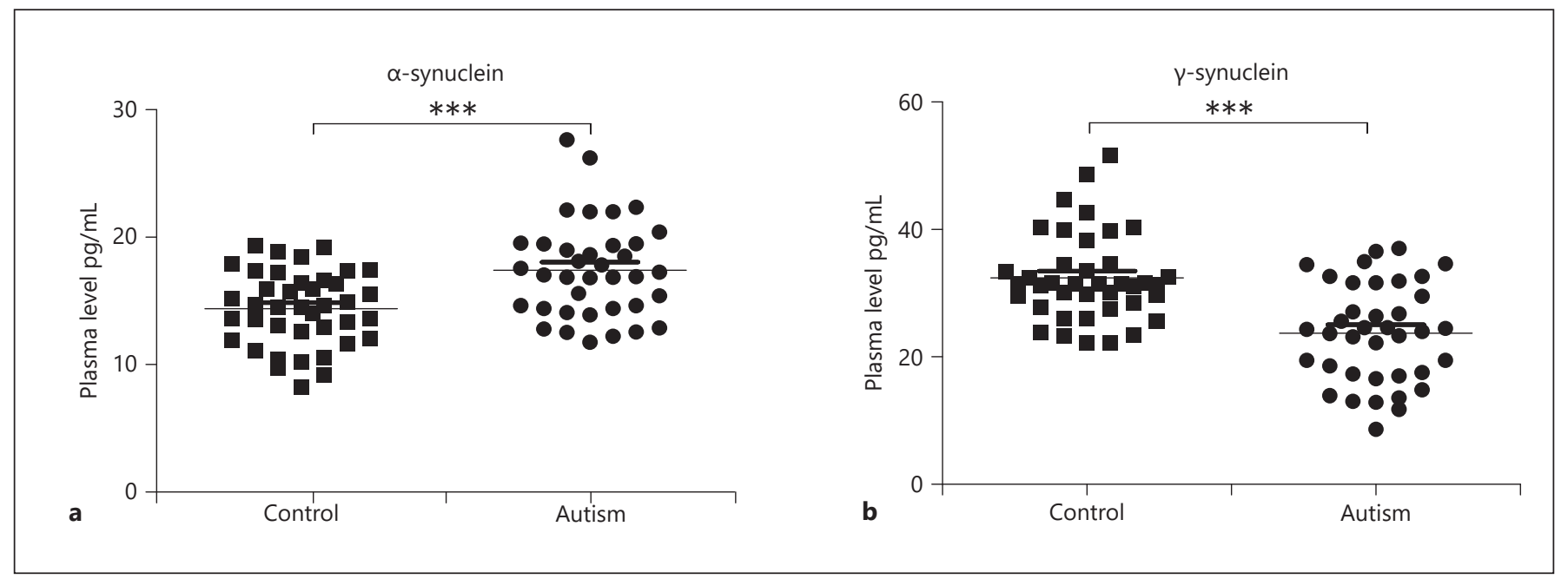

Fig. 1. Plasma levels of $a$-synuclein and $\gamma$-synuclein in the ASD group and control group. a Plasma level of $\mathbf{a}$-synuclein in the ASD group was significantly higher than in the control group. $\mathbf{b}$ Plasma level of $\gamma$-synuclein in the ASD group was significantly lower than in the control group. ${ }^{*} p<0.05 ;{ }^{* *} p<0.01 ;{ }^{* * *} p<0.001$. ASD, autism spectrum disorder.

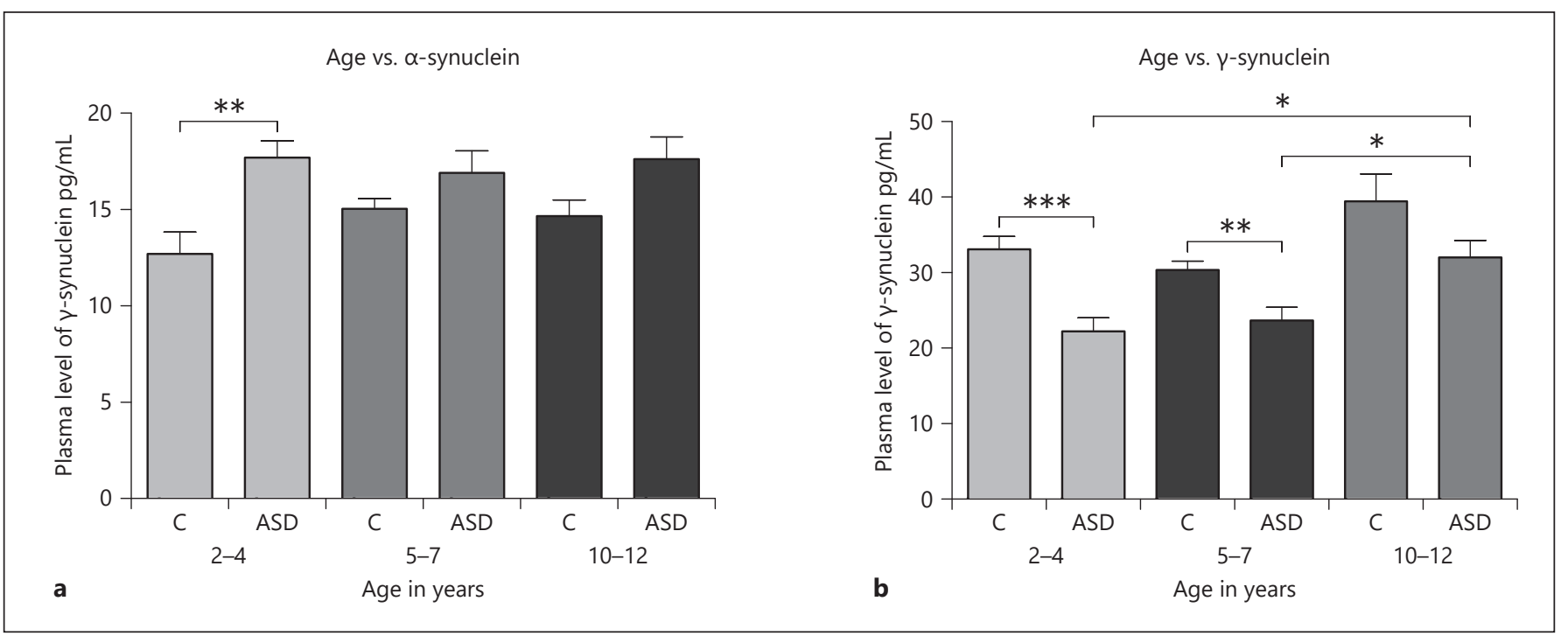

Fig. 2. a, b Change in plasma levels of $\alpha$-synuclein and $\gamma$-synuclein in the ASD group with different age subgroups. a Plasma level of $\alpha$-synuclein in the ASD group in different age subgroups compared to their age-matched control groups. $\mathbf{b}$ Plasma level of $\gamma$-synuclein in the ASD group in different age subgroups compared to their agematched control groups. ${ }^{*} p<0.05 ;{ }^{* *} p<0.01 ;{ }^{* *} p<0.001$. C, control group, ASD, autism spectrum disorder group.

to the severity of ASD, the ASD group was divided into subgroups according to their CARS, SRS, and SSP scores.

CARS analysis showed that $68.4 \%$ of the ASD patients had moderate autism, while $31.8 \%$ had severe autism. There was a significant increase in plasma levels of

Plasma Levels of Alpha and Gamma Synucleins in Autism a-synuclein in the mild $(p<0.01)$ and severe $(p<0.001)$ ASD subgroups compared to the control group. No significant difference was observed between the severe ASD and moderate ASD subgroups. Also, there was a significant decrease in plasma levels of $\gamma$-synuclein in both mild and severe ASD subgroups compared to the control group 

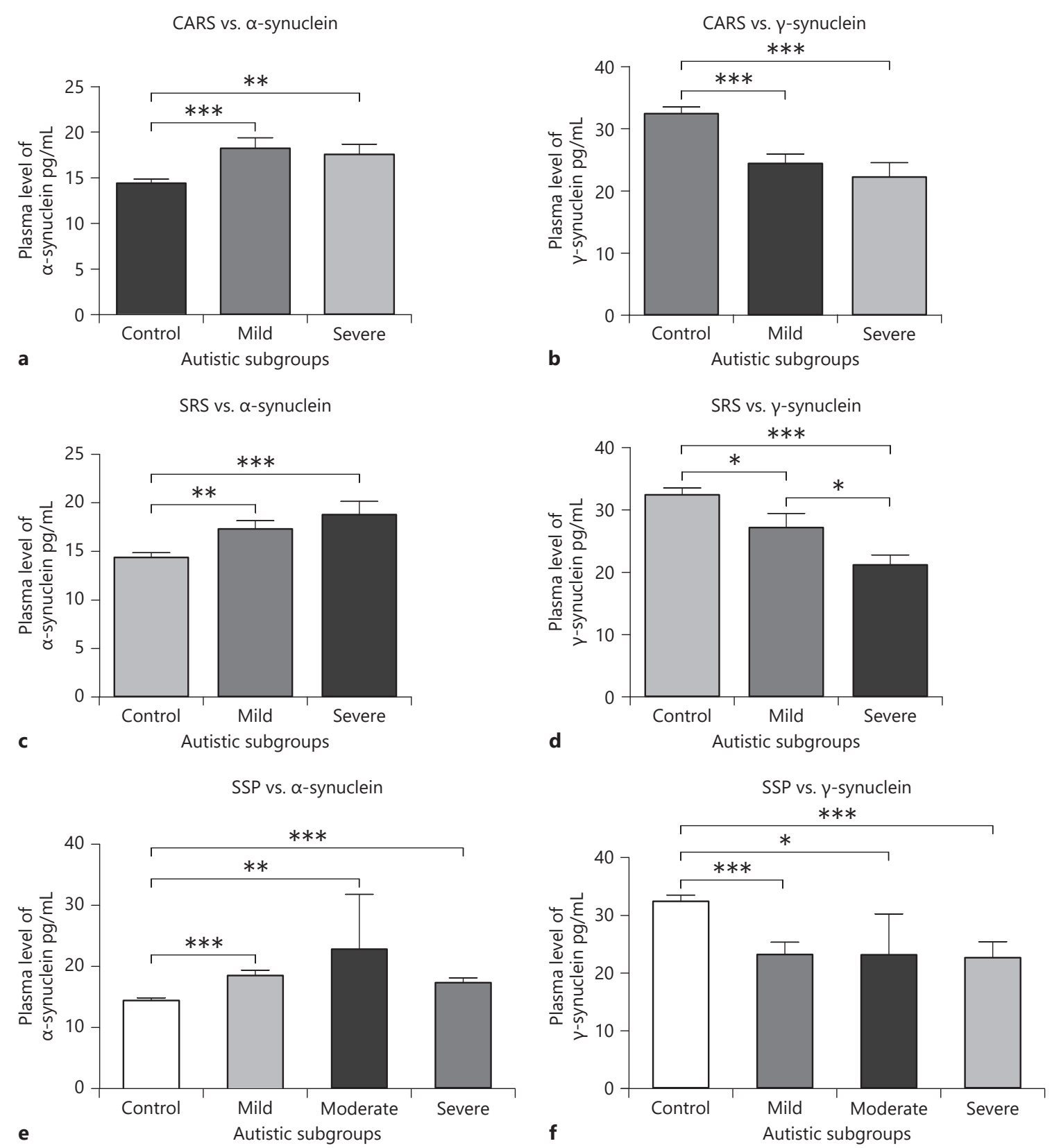

Fig. 3. a-f Correlating plasma levels of $\alpha$-synuclein and $\gamma$-synuclein in ASD subgroups. a-c Correlating plasma levels of a-synuclein with CARS, SRS, and SSP scores in ASD subgroups and the control group. d-f Correlating plasma levels of $\gamma$-synuclein with CARS, SRS, and SSP scores in ASD subgroups and the control group. ${ }^{*} p<0.05$; ${ }^{* *} p<0.01 ;{ }^{* * *} p<0.001$. ASD, autism spectrum disorder; CARS, childhood autism rating score; SSP, short sensory profile; SRS, social responsiveness scale.

$(p<0.001)$. There was no significant correlation found between the ASD subgroups (Fig. 3a, b).

Our data analysis of the SRS showed that $57.9 \%$ of the ASD group had severe autistic behavior, and $42.1 \%$ had mild autistic behavior. Compared to the control group, plasma levels of $\alpha$-synuclein in mild and severe SRS in the ASD group were significantly higher $(p<0.01, p<0.001)$, respectively. No significant difference was observed be- 
tween the ASD subgroups. Levels of $\gamma$-synuclein in the severe ASD subgroup were significantly decreased compared to the control group $(p<0.001)$ and in the mild ASD subgroup compared to controls $(p<0.05)$ (Fig. 3c, d). Also, there was a significant decrease in the severe ASD subgroup compared to the mild ASD subgroup $(p<0.05)$.

The severity of sensory impairment in ASD patients was assessed by SSP analysis. The ASD group showed that $71 \%$ of the patients had a severe sensory impairment, $7.9 \%$ had a moderate sensory impairment, and $21 \%$ had a mild sensory impairment. Plasma levels of $\alpha$-synuclein in all the ASD subgroups (mild, moderate, and severe) were significantly higher than the control group $(p<$ $0.001, p<0.01, p<0.001$ ), respectively. Also, there was a significant decrease in plasma levels of $\gamma$-synuclein in ASD subgroups compared to the control group $(p<0.001$, $p<0.05, p<0.0001$ ), respectively, but no correlation was observed between the ASD subgroups and levels of $\alpha$-synuclein and $\gamma$-synuclein (Fig. 3e, f).

Our data indicate that plasma levels of both $\alpha$-synuclein and $\gamma$-synuclein are significantly associated with the severity of ASD. Although both proteins are correlated to the severity in between ASD subgroups, only plasma levels of $\gamma$-synuclein in the ASD subgroups (mild compared to severe subgroup) are found to be significantly correlated with social impairment measured by the SRS $(p<0.05)$.

\section{Discussion}

The present study is the first to report the association of both $\alpha$-synuclein and $\gamma$-synuclein with the severity of ASD compared to healthy controls. Although $\alpha$-synuclein is an intracellular protein that is mostly located in the brain, several studies have reported its presence in the plasma as well as in the cerebrospinal fluid (CSF) [12]. Levels of $\alpha$-synuclein were significantly higher in ASD patients than in healthy controls. This result refutes 2 previous studies that reported a decrease in $\alpha$-synuclein levels in ASD patients compared to controls. In these studies, the sample size was less than our study and included both sexes [13] and other types of ASD [14]. Although sex and age might not affect the results in the age-groups we studied, we included only males and subcategorized the plasma levels of $\alpha$-synuclein and $\gamma$-synuclein according to the age and included only autistic children. This is because previous studies showed that $\alpha$-synuclein was significantly affected by the age and sex in patients with PD $[15,16]$. Many studies have suggested that $\alpha$-synuclein as a biological biomarker for $\mathrm{PD}, \mathrm{AD}$, and cognitive impairment.
Previous studies have reported an increased level of a-synuclein in neurodegenerative diseases, especially in PD and mild cognitive impairment [12]. Other reports showed that $\alpha$-synuclein is decreased or similar in PD patients compared to controls and did not support $\alpha$-synuclein as a reliable biomarker of $\mathrm{PD}, \mathrm{AD}$, and DLBs [17]. As the concentration of plasma $\alpha$-synuclein is higher than those measured in CSF of PD patients, the increased plasma levels of $\alpha$-synuclein in the present study might be affected by the $\alpha$-synuclein in the peripheral blood [12]. Thus, measurement of $\alpha$-synuclein in the CSF would yield more accurate results, but this procedure is invasive and not ideal for routine monitoring. Another reason for the elevated plasma $\alpha$-synuclein in our study might be due to the clearance of excess $\alpha$-synuclein from the brain leading to the efflux of $\alpha$-synuclein from the central nervous system into the extracellular environment [18]. Also, oxidative stress, which is found to be increased in ASD patients, induces $\alpha$-synuclein aggregation in PD patients and other neurodegenerative diseases and was found to be an important factor in disease initiation and progression [19, 20]. In addition, $\alpha$-synuclein is involved in the regulation of synaptic plasticity which causes synaptic dysfunction, neurotransmitter disturbances, and affects dopamine homeostasis [21]. It might also lead to neuronal death. Thus, this might be related to the synaptic dysfunction that has been reported in ASD patients and dopaminergic genes and pathways disturbances that are found to be strongly associated with ASD pathogenesis [3, 22].

In the present study, both $\alpha$-synuclein and $\gamma$-synuclein were significantly associated with ASD patients compared to control subjects. Similar results were reported from a genetic study on PD and DLB patients [7]. Also, it has been reported that both $\alpha$-synuclein and $\gamma$-synuclein affect synaptic transmission and are associated with neurodegenerative diseases. Some studies have reported that $\gamma$-synuclein dysfunction is correlated with neurodegenerative diseases. Some of these studies reported overexpression of $\gamma$-synuclein in neurodegenerative diseases, while others reported that $\gamma$-synuclein has a neuroprotective role and lower levels are associated with neurodegeneration $[23,24]$.

$\gamma$-Synuclein is a soluble protein that has an important role in synaptic functions and neural growth [4]. It also facilitates the expression and release of BDNF which has a neuroprotective effect and was found to be decreased in neurodegenerative diseases [25]. A study reported that $\mathrm{BDNF}$ is associated with the severity of neurobehavioral deficit [26]. Also, $\gamma$-synuclein is needed for the progression of astrocytes through the cell cycle, and disruption of 
$\gamma$-synuclein function leads to astrocyte apoptosis, possibly leading to neurological diseases [25]. In the present study, $\gamma$-synuclein was significantly lower in ASD patients than in controls, which might cause a disruption of neuroprotective functions of $\gamma$-synuclein in ASD patients.

Our findings indicated that there is an association between plasma levels of both $\alpha$-synuclein and $\gamma$-synuclein and the severity of ASD compared to the control group as shown in Figure 3a-f. Previous studies also reported a correlation between both $\alpha$-synuclein or $\gamma$-synuclein in memory, cognitive functions, and social communication [10, 27]. The association between intellectual disabilities and synaptic dysfunction in autistic children was found to be significant [28]. In our study, the correlation between the severity of ASD and the plasma levels of synaptic proteins ( $\alpha$-synuclein or $\gamma$-synuclein) might also be caused by the disruption of the synaptic functions in ASD patients.

\section{Conclusion}

Abnormal plasma levels of $\alpha$-synuclein and/or $\gamma$-synuclein might be implicated in the synaptic or neuronal pathogenesis of ASD. Further studies on larger sample sizes are required to determine whether $a$-synuclein and/or $\gamma$-synuclein leads to synaptic or neural disruption, both of which occur in ASD patients. Also, these proteins could be a potential therapeutic target for neurobehavioral deficit in ASD patients or a potential target for early diagnosis and treatment of ASD.

\section{Acknowledgements}

This project was funded by the National Plan for Science, Technology and Innovation (MAARIFAH), King AbdulAziz City for Science and Technology, Kingdom of Saudi Arabia (award number: 08-MED 510-02).

\section{Statement of Ethics}

The study was approved by the Institutional Review Board (IRB) of college of medicine research center at King Saud University. Funding.

\section{Conflict of Interest Statement}

The authors report having no biomedical financial interests. This disclosure includes direct or indirect financial or personal relationships, interests, and affiliations relevant to the subject matter of the manuscript that have occurred over the last 2 years or that are expected in the foreseeable future. This disclosure includes, but is not limited to, grants or funding, employment, affiliations, patents (in preparation, filed, or granted), inventions, honoraria, consultancies, royalties, stock options/ownership, or expert testimony. Also, the authors have no conflicts of interest to declare.

\section{Funding Sources}

This project was funded by the National Plan for Science, Technology and Innovation (MAARIFAH), King AbdulAziz City for Science and Technology, Kingdom of Saudi Arabia Award Number (08-MED 510 -02).

\section{References}

1 Emberti Gialloreti L, Mazzone L, Benvenuto A, Fasano A, Alcon AG, Kraneveld A, et al. Risk and protective environmental factors associated with autism spectrum disorder: evidence-based principles and recommendations. J Clin Med. 2019;8(2):217.

2 Chiarotti F, Venerosi A. Epidemiology of autism spectrum disorders: a review of worldwide prevalence estimates since 2014. Brain Sci. 2020;10(5):10.

3 Chen J, Yu S, Fu Y, Li X. Synaptic proteins and receptors defects in autism spectrum disorders. Front Cell Neurosci. 2014;8:276.

4 Vargas KJ, Schrod N, Davis T, FernandezBusnadiego R, Taguchi YV, Laugks U, et al. Synucleins have multiple effects on presynaptic architecture. Cell Rep. 2017;18(1):161-73.

5 Jain MK, Singh P, Roy S, Bhat R. Comparative analysis of the conformation, aggregation, interaction, and fibril morphologies of human $\alpha-, \beta$-, and $\gamma$-synuclein proteins. Biochemistry. 2018;57(26):3830-48.
6 Zhang G, Xia Y, Wan F, Ma K, Guo X, Kou L, et al. New perspectives on roles of alpha-synuclein in Parkinson's disease. Front Aging Neurosci. 2018;10:370.

7 Nishioka K, Wider C, Vilariño-Güell C, SotoOrtolaza AI, Lincoln SJ, Kachergus JM, et al. Association of alpha-, beta-, and gammasynuclein with diffuse lewy body disease. Arch Neurol. 2010;67(8):970-5.

8 Kang SS, Zhang Z, Liu X, Manfredsson FP, Benskey MJ, Cao X, et al. TrkB neurotrophic activities are blocked by $\alpha$-synuclein, triggering dopaminergic cell death in Parkinson's disease. Proc Natl Acad Sci U S A. 2017; 114(40):10773-8.

9 Clinton LK, Blurton-Jones M, Myczek K, Trojanowski JQ, LaFerla FM. Synergistic Interactions between Abeta, tau, and alphasynuclein: acceleration of neuropathology and cognitive decline. J Neurosci. 2010; 30(21):7281-9.
10 Kokhan VS, Kokhan TYG, Samsonova AN, Fisenko VP, Ustyugov AA, Aliev G. The dopaminergic dysfunction and altered working memory performance of aging mice lacking gamma-synuclein gene. CNS Neurol Disord Drug Targets. 2018;17(8):604-7.

11 Morato Torres CA, Wassouf Z, Zafar F, Sastre D, Outeiro TF, Schüle B. The role of alpha-synuclein and other Parkinson's genes in neurodevelopmental and neurodegenerative disorders. Int J Mol Sci. 2020;21(16): 5724.

12 Seino Y, Nakamura T, Kawarabayashi T, Hirohata $\mathrm{M}$, Narita S, Wakasaya Y, et al. Cerebrospinal fluid and plasma biomarkers in neurodegenerative diseases. J Alzheimers Dis. 2019;68(1):395-404.

13 Kadak MT, Cetin I, Tarakçığlu MC, Özer ÖF, Kaçar S, Çimen B. Low serum level a-synuclein and tau protein in autism spectrum disorder compared to controls. Neuropediatrics. 2015;46(6):410-5. 
14 Sriwimol W, Limprasert P. Significant changes in plasma alpha-synuclein and beta-synuclein levels in male children with autism spectrum disorder. Biomed Res Int. 2018;2018: 4503871.

15 Caranci G, Piscopo P, Rivabene R, Traficante A, Riozzi B, Castellano AE, et al. Gender differences in Parkinson's disease: focus on plasma alpha-synuclein. J Neural Transm. 2013; 120:1209-15.

16 Brighina L, Prigione A, Begni B, Galbussera A, Andreoni S, Piolti R, et al. Lymphomonocyte alpha-synuclein levels in aging and in Parkinson disease. Neurobiol Aging. 2010; 31(5):884-5.

17 Öhrfelt A, Grognet P, Andreasen N, Wallin A, Vanmechelen E, Blennow K, et al. Cerebrospinal fluid alpha-synuclein in neurodegenerative disorders-a marker of synapse loss? Neurosci Lett. 2009;450(3):332-5.

18 Lopes da Fonseca T, Villar-Piqué A, Outeiro TF. The interplay between alpha-synuclein clearance and spreading. Biomolecules. 2015; 5(2):435-71.
19 Bjørklund G, Meguid NA, El-Bana MA, Tinkov AA, Saad K, Dadar M, et al. Oxidative stress in autism spectrum disorder. $\mathrm{Mol} \mathrm{Neu-}$ robiol. 2020;57(5):2314-32.

20 Paxinou E, Chen Q, Weisse M, Giasson BI, Norris EH, Rueter SM, et al. Induction of alpha-synuclein aggregation by intracellular nitrative insult. J Neurosci. 2001;21(20):805361.

21 Nemani VM, Lu W, Berge V, Nakamura K, Onoa B, Lee MK, et al. Increased expression of alpha-synuclein reduces neurotransmitter release by inhibiting synaptic vesicle reclustering after endocytosis. Neuron. 2010;65(1): 66-79.

22 Nguyen M, Roth A, Kyzar EJ, Poudel MK, Wong K, Stewart AM, et al. Decoding the contribution of dopaminergic genes and pathways to autism spectrum disorder (ASD). Neurochem Int. 2014;66:15-26.

23 Wilding C, Bell K, Beck S, Funke S, Pfeiffer N, Grus FH. $\gamma$-Synuclein antibodies have neuroprotective potential on neuroretinal cells via proteins of the mitochondrial apoptosis pathway. PLoS One. 2014;9(3):e90737.
24 Ninkina N, Peters O, Millership S, Salem H, van der Putten $\mathrm{H}$, Buchman VL. Gammasynucleinopathy: neurodegeneration associated with overexpression of the mouse protein. Hum Mol Genet. 2009;18(10):1779-94.

25 Winham CL, Le T, Jellison ER, Silver AC, Levesque AA, Koob AO. $\gamma$-synuclein induces human cortical astrocyte proliferation and subsequent BDNF expression and release. Neuroscience. 2019;410:41-54.

26 Kasarpalkar NJ, Kothari ST, Dave UP. Brainderived neurotrophic factor in children with autism spectrum disorder. Ann Neurosci. 2014;21(4):129-33.

27 Yang N, Li Z, Han D, Mi X, Tian M, Liu T, et al. Autophagy prevents hippocampal a-synuclein oligomerization and early cognitive dysfunction after anesthesia/surgery in aged rats. Aging. 2020;12(8):7262-81.

28 Zoghbi HY, Bear MF. Synaptic dysfunction in neurodevelopmental disorders associated with autism and intellectual disabilities. Cold Spring Harb Perspect Biol. 2012;4(3):a009886. 